\title{
Coordinated Dispersion and Aggregation of Gold Nanorod in Aptamer-Mediated Gestational Hypertension Analysis
}

\author{
Xiucui Bao $\mathbb{D}^{1},{ }^{1}$ Gaoxiang Huo, ${ }^{1}$ Li Li, ${ }^{1}$ Xuebin Cao, ${ }^{2}$ Yamei Liu, ${ }^{1}$ Thangavel Lakshmipriya, ${ }^{3}$ \\ Yeng Chen, ${ }^{4}$ Firdaus Hariri, ${ }^{5}$ and Subash C. B. Gopinath $\mathbb{D}^{3,6}$ \\ ${ }^{1}$ Department of Obstetrics, Yihe Maternity District of Cangzhou People's Hospital, Cangzhou, Hebei 061000, China \\ ${ }^{2}$ Department of General Surgery, Cangxian Hospital, Cangzhou, Hebei 061000, China \\ ${ }^{3}$ Institute of Nano Electronic Engineering, Universiti Malaysia Perlis, 01000 Kangar, Perlis, Malaysia \\ ${ }^{4}$ Department of Oral \& Craniofacial Sciences, Faculty of Dentistry, University of Malaya, 50603 Kuala Lumpur, Malaysia \\ ${ }^{5}$ Department of Oral and Maxillofacial Clinical Sciences, Faculty of Dentistry, University of Malaya, \\ 50603 Kuala Lumpur, Malaysia \\ ${ }^{6}$ School of Bioprocess Engineering, Universiti Malaysia Perlis, 02600 Arau, Perlis, Malaysia
}

Correspondence should be addressed to Xiucui Bao; baoxiucui@sina.com

Received 4 April 2019; Revised 1 June 2019; Accepted 18 June 2019; Published 11 November 2019

Guest Editor: Xiang Li

Copyright (c) 2019 Xiucui Bao et al. This is an open access article distributed under the Creative Commons Attribution License, which permits unrestricted use, distribution, and reproduction in any medium, provided the original work is properly cited.

Gestational hypertension is one of the complicated disorders during pregnancy; it causes the significant risks, such as placental abruption, neonatal deaths, and maternal deaths. Hypertension is also responsible for the metabolic and cardiovascular issues to the mother after the years of pregnancy. Identifying and treating gestational hypertension during pregnancy by a suitable biomarker is mandatory for the healthy mother and foetus development. Cortisol has been found as a steroid hormone that is secreted by the adrenal gland and plays a pivotal role in gestational hypertension. A normal circulating level of cortisol is involved in the regulation of blood pressure, and it is necessary to monitor the changes in the level of cortisol during pregnancy. In this work, aptamer-based colorimetric assay is demonstrated as a model with gold nanorod to quantify the level of cortisol using the coordinated aggregation (at $500 \mathrm{mM}$ of $\mathrm{NaCl}$ ) and dispersion (with $10 \mu \mathrm{M}$ of aptamer), evidenced by the scanning electron microscopy observation and UV-visible spectroscopy analysis. This colorimetric assay is an easier visual detection and reached the limit of detection of cortisol at $0.25 \mathrm{mg} / \mathrm{mL}$. This method is reliable to identify the condition of gestational hypertension during the pregnancy period.

\section{Introduction}

Gestational hypertension or pregnancy-induced hypertension complicates $\sim 10 \%$ of the pregnant cases and causes a poor perinatal outcome. It is also responsible for raising other diseases, such as elevated blood pressure in the artery, preeclampsia, and eclampsia, during the period of pregnancy $[1,2]$. In addition, there is a possibility of affecting other parts in the body, such as kidney and heart, and inducing an early delivery. In general, gestational hypertension arises during the second half of pregnancy. Identifying hypertension by a suitable biomarker is mandatory for a healthy pregnant woman [3]. Cortisol is a stress hormone that is secreted from the adrenal gland. It spikes into the main stream of the body during the time of high stress and elevates the cortisol level in the bloodstream. It has been proved that the serum cortisol plays a major role in the pathophysiology of the gestational hypertension [4], especially the higher level of cortisol causes hypertension and endothelial dysfunction

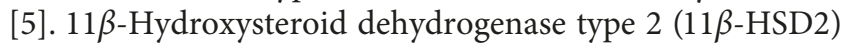
is an enzyme, produced in the renal tubules; it converts cortisol into an inactive cortisone, thus permitting the mineralocorticoid (a receptor) as aldosterone-selective. The functional diminishes of this enzyme cause the mutations with HSD11B2 gene, which encodes $11 \beta$-HSD2, mainly considered as an initiative for hypertension [6]. Therefore, 
measuring the level of cortisol of the pregnant women during the trimester is considered to be important for estimating the function of $11 \beta$-HSD2 [7].

In the current investigation, an aptamer-based colorimetric assay was performed to quantify the level of cortisol. Aptamer, a DNA or RNA molecule, has been generated from the randomized library of molecules by a method "SELEX" (Systematic Evaluation of Ligands by Exponential enrichment) with three vital steps, which includes binding, separation, and amplification [8-11]. Since aptamers carry the advantages over antibodies, such as easier to synthesize, cheaper, amenable to the modifications, high affinity, and nonimmunogenic, various aptamers were generated against a wide range of targets from the lower-molecular weight molecules to the intact cell. The generated aptamers have been applied in different fields such as medical, environmental, drug delivery, imaging, and biosensors. Due to the highly selective and sensitive binding nature of aptamer to its target molecule, it has been widely applied in the field of biosensors and more prevalent to diagnose various diseases at a higher affinity. Aptamers that were demonstrated with various biosensors, which include surface plasmon resonance [12, 13], waveguide mode sensor [14], colorimetric [15], and RAMAN spectroscopy [16], help to detect diseases from the basic viral infection to death-causing diseases, such as cancer [17-20]. Among the revealed sensors, colorimetric analysis with aptamers brings out several positive features, such as easier visualization, rapidness, cheaper, effective, and used to detect tiny analytes including heavy metals [21], smaller molecular weight proteins [22], DNA [23], and cancer biomarkers [24], without involving sophisticated instrumentation and trained personnel [25].

The visual colorimetric analysis is the salt-induced aggregation assay by utilizing DNA, RNA, or aptamers with the gold nanostructure to detect the desired target. Gold is one of the unavoidable materials in the field of biosensors due to its versatile physical and chemical properties. Moreover, gold nanoparticle (GNP) is smaller, suitable to confine the electrons in order to produce the quantum effects, a key consideration for the colorimetric assay [26]. In addition, the functionalized GNP leads to find several downstream applications. Due to the abovementioned positive features, gold nanomaterial and the gold surface have been applied efficiently in all types of sensor to detect different biomarkers [8, 27-30]. In general, the unmodified dispersed GNPs have a bright red-wine color and changes its color to purple or blue when it aggregates under ionic condition $[30,31]$. This controlled change in color induced by the aggregation can be the basis of colorimetric assay. In the case of aptamer-based colorimetric assay, aptamers are immobilized on the surface of the gold through the electrostatic attraction [15]. When the aptamer is bound to the target, the color of the GNP solution changed to purple at a high salt concentration. This study has utilized a modified gold nanorod (GNR) attached with anticortisol aptamer to interact with the cortisol (target), a model system that can be applied to measure the gestational hypertension by quantifying the cortisol as in earlier study [32].

\section{Materials and Methods}

2.1. Materials. Gold nanorod (GNR) was obtained from Nanocs, USA. Sodium chloride $(\mathrm{NaCl})$ was procured from Sigma-Aldrich, USA. The hormones cortisol and progesterone were from Adooq Biosciences (USA). Norepinephrine was from Abcam (USA). Anticortisol sequence was adapted from Sanghavi et al. [32] and synthesized commercially. Buffers and other reagents were obtained in pure form and used directly. The size and shape of the GNR were observed under field-emission scanning electron microscopy at $500 \mathrm{~nm}$ scale.

\subsection{Optimization of Monovalent Ions on Gold Nanorod for} Salt-Induced Aggregation. To perform the colorimetric assay, first optimize a suitable concentration of $\mathrm{NaCl}$ to induce the aggregation of GNR. Different concentrations of $\mathrm{NaCl}$ were added independently with the constant volume of $10 \mu \mathrm{l}$ GNR (final concentrations were 15, 30, 60, 125, 250, and $500 \mathrm{mM}$ ) and kept for $10 \mathrm{~min}$ at room temperature. The changes in the colors were noticed and the maximum wavelength absorbance was measured by using the UVvisible spectrophotometer, in which the scanned wavelength ranged from 400 to $750 \mathrm{~nm}$.

\subsection{Optimization of Aptamer Attachment on Gold Nanorod.} Before performing the colorimetric assay, the condition was optimized for the right aptamer concentration to stabilize the GNR at a high salt concentration. Different concentrations of the diluted aptamer were mixed with $10 \mu \mathrm{l}$ of GNR (final concentration will be $1.25,2.5,5,10,15$, and $20 \mu \mathrm{M}$ ) independently and kept for $30 \mathrm{~min}$ at RT. After that, the optimal higher concentration of $\mathrm{NaCl}$ was added to each dilution and incubated for $10 \mathrm{~min}$ to observe the changes with the color of GNR. The changes in the colors were noticed and the absorbance wavelength maximum was measured by using the UV-visible spectrophotometer scanned from 400 to $750 \mathrm{~nm}$.

\subsection{Colorimetric Detection of Cortisol Using Anticortisol} Aptamer on GNR. The aptamer modified GNR (aptamerGNR) was used to detect the cortisol. For that, $1 \mathrm{mg} / \mathrm{mL}$ of cortisol was added with aptamer-GNR and kept for $30 \mathrm{~min}$ at RT. Then, the higher concentration of $\mathrm{NaCl}$ was added to the solution to observe the color change. After the confirmation of detection, to evaluate the limit of detection, the cortisol concentrations were titrated from 0.625 to $1 \mathrm{mg} / \mathrm{mL}$ by interacting aptamer-GNR. Specific detection of cortisol was carried out with two control hormones, namely norepinephrine and progesterone. For that, $1 \mathrm{mg} / \mathrm{mL}$ of control hormone was mixed with aptamer-GNR and incubated for $30 \mathrm{~min}$ at RT. Then, $\mathrm{NaCl}$ was added to evaluate the interaction of aptamer with control hormones. The results 
obtained were compared with $1 \mathrm{mg} / \mathrm{mL}$ of cortisol interaction with aptamer-GNR.

\section{Results and Discussion}

Gestational hypertension is a critical disorder during the pregnancy period and it causes various issues with foetus development and delivery. Finding a level of hypertension is necessary to take care of the mother and baby during and after the period of pregnancy. Cortisol is the stress hormone and its level plays a crucial role in causing different diseases, such as gestational hypertension, during pregnancy.

In the materials study, it has been widely accepted that the wavelength shift of the plasmon band with the gold shows a big impact in the biosensing applications. In general, the spherical-shaped gold particles have been used for the colorimetric assay to induce a large shift for the high-performance detection, in which the controlled aggregation and dispersion causes the spectral difference and in the presence of the target, aggregation with ionic solution displays a broad spectrum under UV-visible spectroscopy scanning. However, GNP-based colorimetric assay is not suitable for multiplex analysis due to the absence of a properly shaped spectrum. Researchers are looking for an alternate particle to minimize a wide spectrum in order to move towards the multiple target analysis. It has been revealed that the usage of anisotropic silver nanoparticles with tetrahedron shows a spectral shift upon target interaction but it does not cause the aggregation, and demonstrated a microarray for molecular fingerprint analysis. Researchers also proposed the usage of GNR to overcome the high aggregation, as GNR can be fabricated at different range of size ratios and has unique advantage for multiplex analysis. Towards this direction, the current study is an attempt to optimize the condition for future multiplex analysis [33]. To support this notion, researchers have demonstrated the multiplex detection based on the plasmon changes by GNR [33].

To proceed in this line, the current research has been carried out to detect the level of cortisol by a gold nanorod(GNR-) based aggregation on colorimetric assay using an aptamer generated against cortisol. Figure 1 shows the schematic representation of the colorimetric assay-based detection of cortisol. As shown in the figure, aptamers are electrostatically bound on the surface of GNR, and upon interacting with cortisol aptamers will be released from GNR. With this condition when adding the higher concentration of $\mathrm{NaCl}$, the GNR will be aggregated and the color of the solution is turned into purple from red (dispersion) (Figure 1(a)). The predicted secondary structure of the tested anticortisol aptamer by mfold software is shown in Figure 1(b), and the aptamer has apparent stems and loops to interact with cortisol.

3.1. Requirement of Optimal Monovalent Ion for GNR Aggregation. Before initiating the detection of cortisol, determination of a suitable concentration of $\mathrm{NaCl}$ is necessary to achieve higher sensitivity; for that, different concentrations of $\mathrm{NaCl}$ were tested with the constant
GNR volume. Figure 2(a) displays the obtained UV-visible spectrum with $\mathrm{NaCl}$ titration on GNR. It is clearly seen that with increase in the $\mathrm{NaCl}$ concentration, the optical density (OD) of the GNR was reduced. With the concentration from 30 to $250 \mathrm{mM}$, the peak position has not been shifted, but at the higher concentration $(500 \mathrm{mM})$, the apparent peak shift was noticed from 550 to $620 \mathrm{~nm}$, due to the aggregation of GNR (Figure 2(b)). The aggregation was evidenced by the field-emission scanning electron microscopy observation (Figure 2(b), inset). This aggregation is due to the $\mathrm{NaCl}$ bridging on the unmodified negatively charged GNRs, resulting in appearance of purple or blue solution [31].

\subsection{Requirement of Optimal Aptamer Concentration for GNR} Dispersion. Upon finding the optimal concentration at $500 \mathrm{mM}$ of $\mathrm{NaCl}$, the experiment was performed to find a suitable concentration of anticortisol aptamer to cover completely the surface of GNR, to be stable under $500 \mathrm{mM}$ of $\mathrm{NaCl}$ in the absence of cortisol. Without the complete coverage, the GNR will cause the aggregation by $\mathrm{NaCl}$ even in the absence of cortisol and leads to the erroneous positive result. For the optimization analysis, initially different concentrations from 1.25 to $10 \mu \mathrm{M}$ of aptamer were mixed independently with the fixed volume of GNR and $500 \mathrm{mM}$ (final concentration) $\mathrm{NaCl}$ was added to check the stability of GNR. In general, thiol-conjugated aptamers have been used to immobilize them on the surface of the gold and they are very stable under a high salt concentration. In our case, we directly immobilized the unmodified aptamer on the surface of the GNR. In principle, the aptamer or single-stranded DNA can attract to the surface of gold nanostructure due to the coordination between gold and "N" atoms in DNA bases. As shown in Figure 3, the aptamer concentration with $1.25 \mu \mathrm{M}$ shows the peak maximum at $620 \mathrm{~nm}$ with the optical absorbance of 0.6 , indicating GNR is in the aggregated form in the presence of $\mathrm{NaCl}$. By increasing the aptamer concentration further, the peak intensity was also increased and all the higher concentrations of aptamer showed the peak at the wavelength $\sim 550 \mathrm{~nm}$, indicating that GNR is in the dispersal state. At the aptamer concentration of $10 \mu \mathrm{M}$, the solution shows the maximum peak intensity with optical absorption as 1.2. This result confirms that the aptamer concentration needed to detect the cortisol ideally by the colorimetric assay is $10 \mu \mathrm{M}$. As shown in Figure 3(a), the concentration at $10 \mu \mathrm{M}$ causes the formation of an apparent peak at $550 \mathrm{~nm}$ with a clear change of the solution to red. To confirm that this concentration is the optimum, we performed the experiments with further concentrations at 15 and $20 \mu \mathrm{M}$. The results clearly displayed that $10 \mu \mathrm{M}$ is the optimum concentration for the current colorimetric assay for the cortisol detection (Figures 3(a) and 3(b)).

3.3. Genuine Interaction of Cortisol and Nonbiofouling. The abovementioned experiments were used to determine the optimal $\mathrm{NaCl}$ and aptamer concentrations. Before proceeding further for the cortisol detection, the nonspecific binding of cortisol on the surface of the GNR was tested. If 


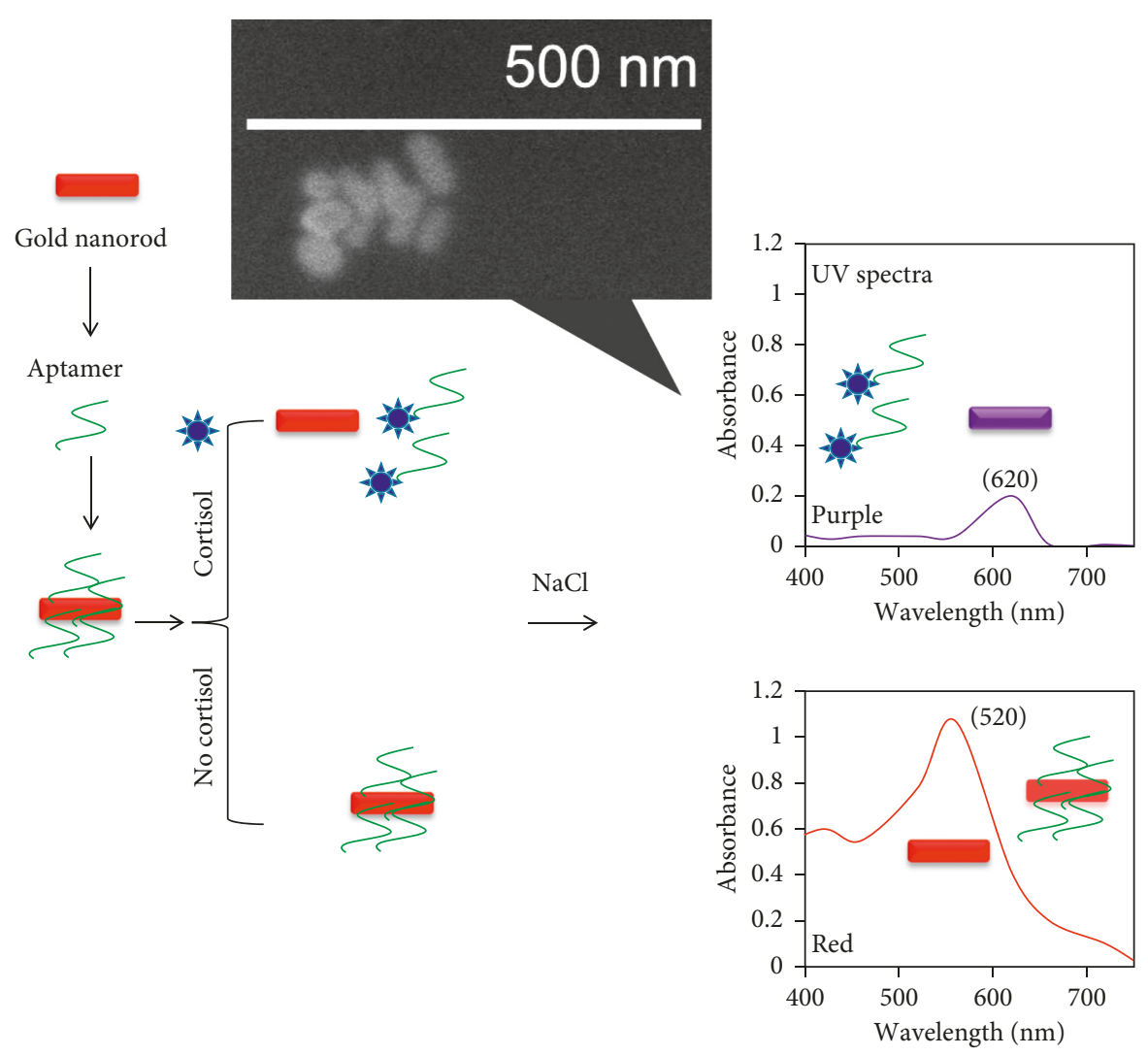

(a)

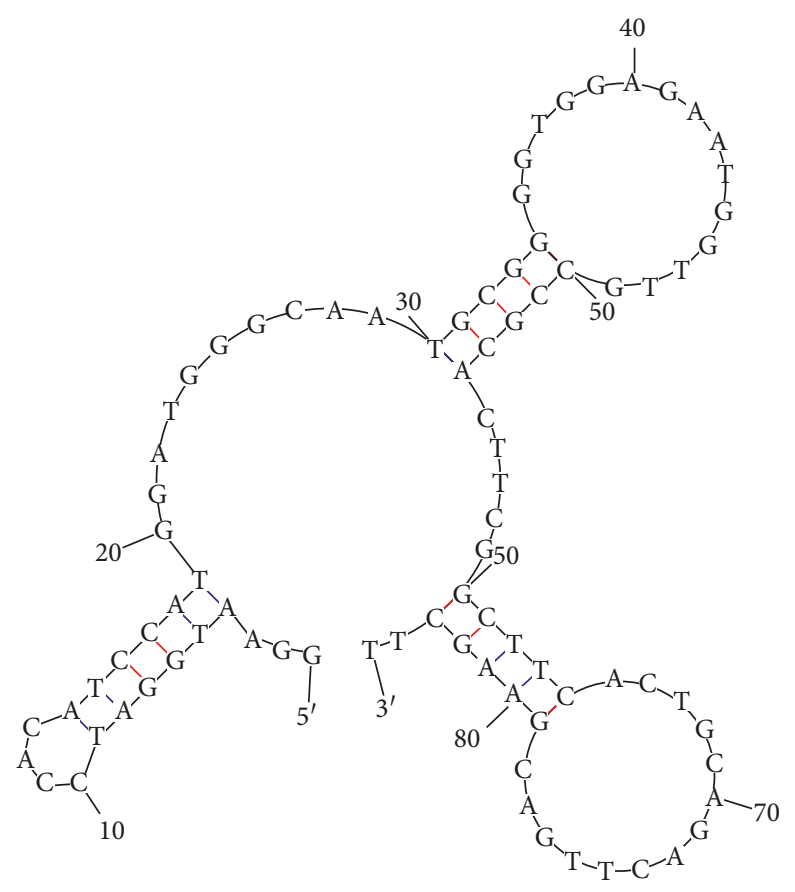

(b)

FIGURE 1: (a) Schematic representation of cortisol detection by aptamer-GNR based colorimetric assay. As-received GNR appears red and in the presence of $\mathrm{NaCl}$, it turned into purple. At higher concentration of aptamer-GNR, it appears to be red even in the presence of $\mathrm{NaCl}$. When aptamer-GNR reacts with cortisol at appropriate concentration, the color of the GNR solution is turned to purple with NaCl, indicating the release of the aptamer from the GNR. The aggregation is displayed by SEM analysis (inset). (b) Secondary structure of anticortisol aptamer. Folded by mfold online software. 


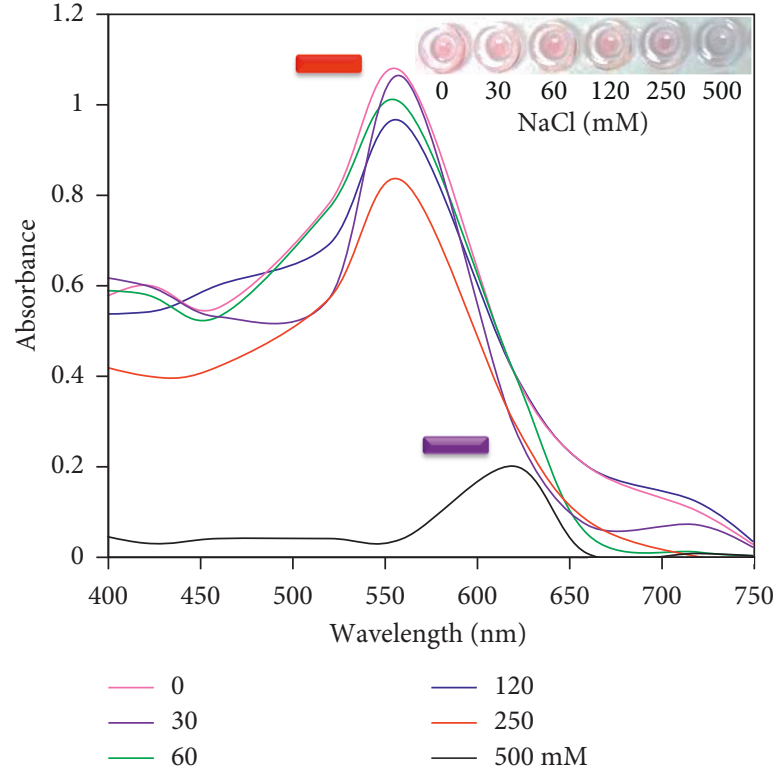

(a)

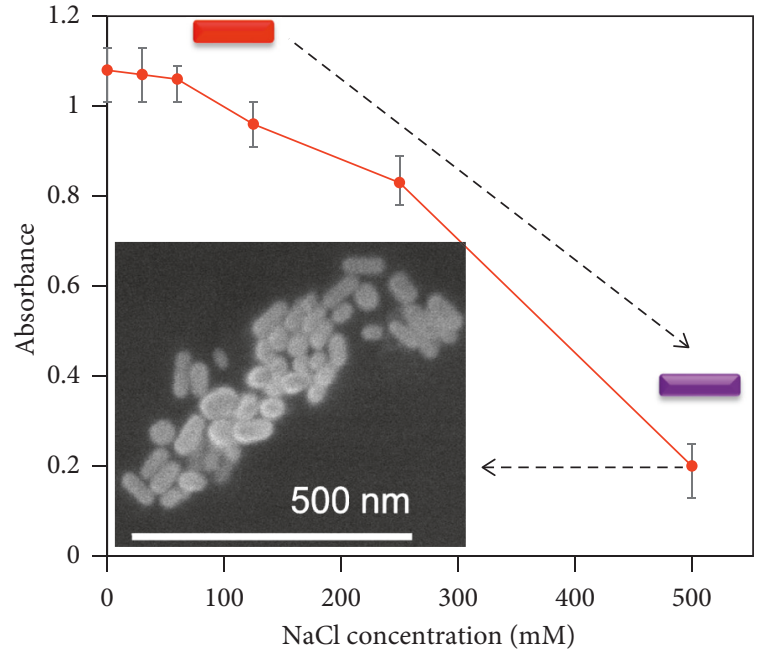

(b)

Figure 2: $\mathrm{NaCl}$ titration on GNR. (a) Different concentrations of (30 to $500 \mathrm{mM}$ ) NaCl were mixed independently with a constant amount of GNR and the aggregation pattern was observed. Inset displays the color developments. (b) Peak absorbance maximum with different concentrations of $\mathrm{NaCl}$, averaged with different experimental replicates. Inset is for aggregation obtained by SEM.

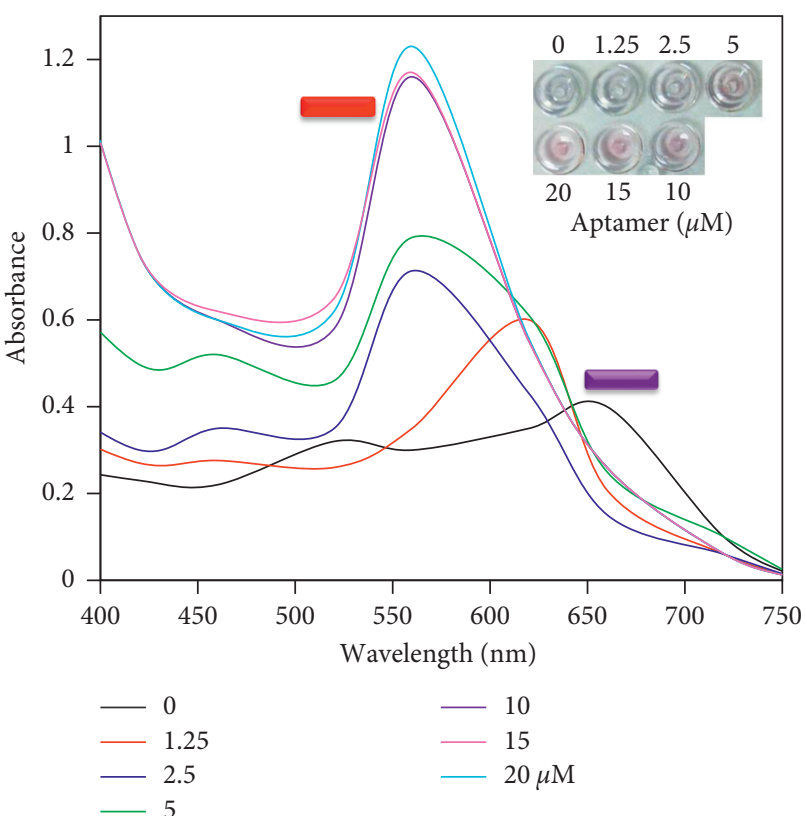

(a)

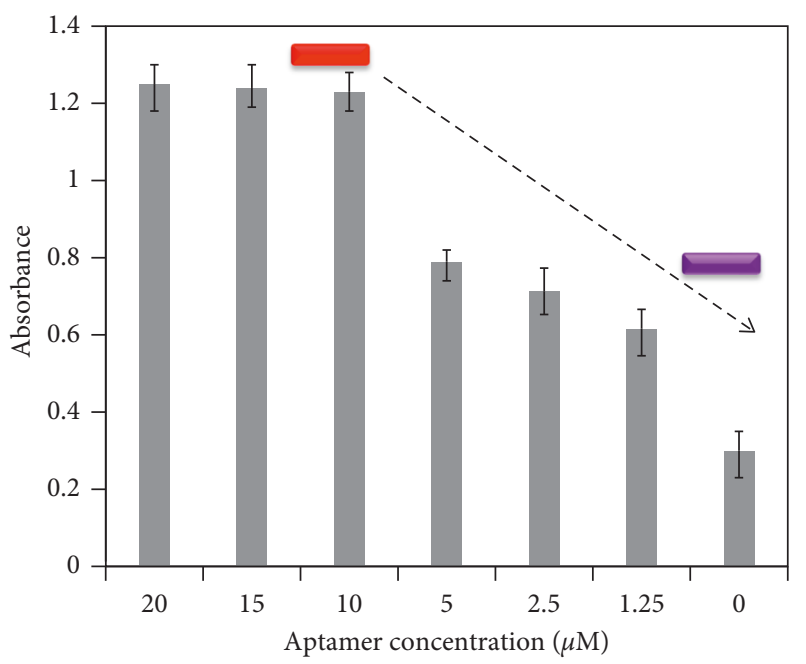

(b)

FIgURE 3: Optimization of aptamer concentration. (a) Aptamer with concentrations of 1.25 to $20 \mu \mathrm{M}$ was mixed independently with GNR and the aggregation was checked in the presence of $\mathrm{NaCl}$. Inset displays the color developments. (b) Peak absorbance maximums with different concentrations of aptamer, averaged with different experimental replicates. The arrow indicates the direction of the changes.

cortisol itself binds on the GNR, it may lead to a falsenegative result. For this analysis, different concentrations of cortisol $(0.5,1,2$, and $4 \mathrm{mg} / \mathrm{mL})$ were mixed independently with the constant GNR and induced the color change by $500 \mathrm{mM}$ of $\mathrm{NaCl}$. It was noticed that even when the concentration of cortisol was increased, the color of the GNR solution turned to purple in the presence of $\mathrm{NaCl}$ due to the aggregation, which means that the cortisol itself is not able to bind on the surface of the GNR, and similar results were observed with all the concentrations tested (Figure 4). 


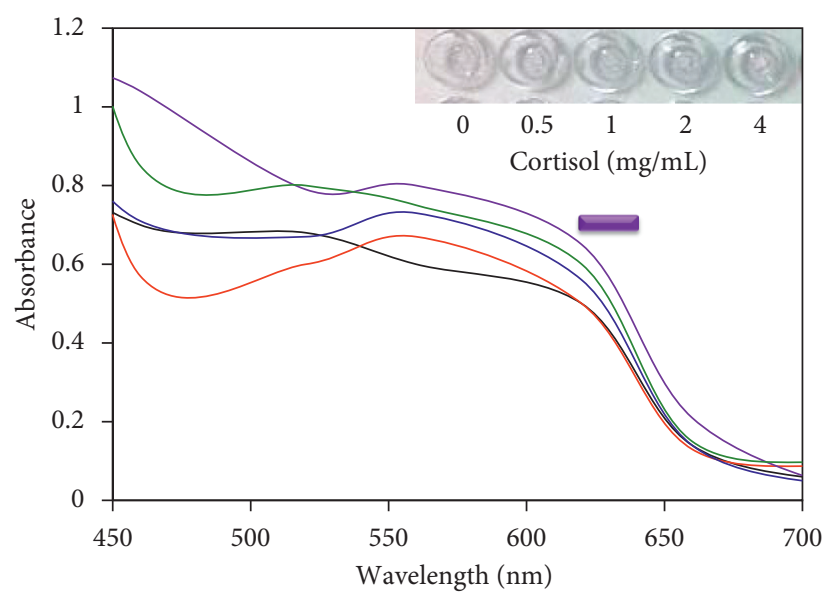

FIgURE 4: Nonfouling effect of cortisol on GNR. Different concentrations of cortisol $(0.5-4 \mathrm{mg} / \mathrm{mL})$ were mixed independently with constant amount of $\mathrm{GNR}$, and $500 \mathrm{mM} \mathrm{NaCl}$ was added to evaluate the nonfouling effect.
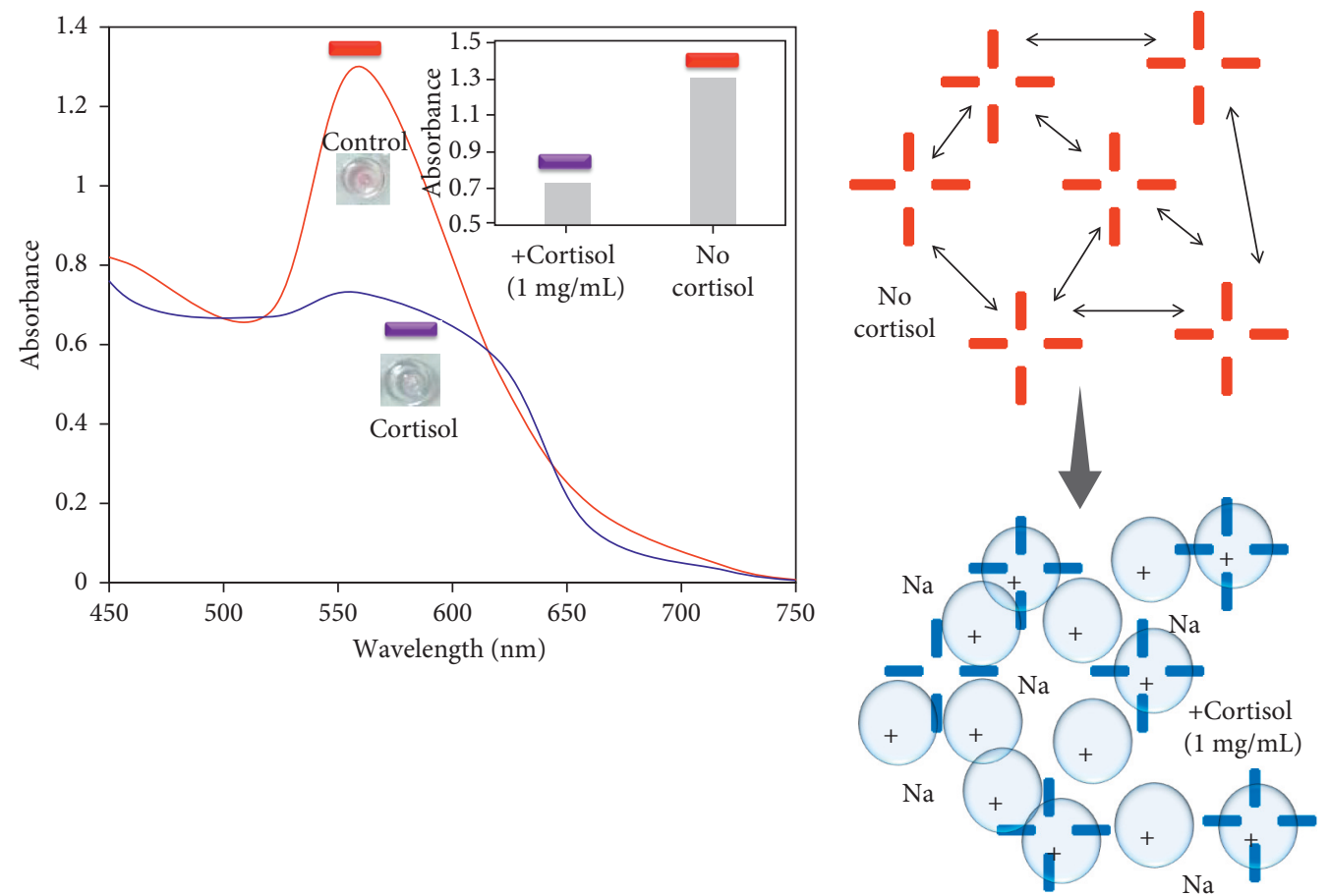

(a)

(b)

FIGURE 5: (a) Detection of cortisol by colorimetric assay on aptamer-GNR conjugates. $1 \mathrm{mg} / \mathrm{mL}$ of cortisol was mixed with aptamer-GNR and checked the aggregation in the presence of $\mathrm{NaCl}$. Inset displays the graphical representation. (b) Mechanism of dispersion and aggregation mimics the above reaction.

3.4. Cortisol in Aggregation of Aptamer-GNR. After all the optimizations, we performed the colorimetric assay using aptamer, cortisol, and GNR with the above final conditions. Initially, the higher concentration $(1 \mathrm{mg} / \mathrm{mL})$ of cortisol was used to evaluate the release of aptamer from the GNR. As shown in Figure 5, in the control experiment using aptamer-GNR (without cortisol), we did not observe the changes with the spectrum at different wavelengths, and it still remains same at the wavelength $550 \mathrm{~nm}$. This means that in the absence of cortisol, the aptamer-GNR kept its red color under a high concentration of salt. At the same time, when we mixed $1 \mathrm{mg} / \mathrm{mL}$ of cortisol to aptamer-GNR, the cortisol interacts with aptamer on GNR and released. Under this condition, at the higher salt concentration, the color of the solution turned into purple and the spectrum was shifted from 550 to $620 \mathrm{~nm}$ due to the aggregation (Figure 5(a) and inset). The apparent mechanism with the aggregation and dispersion is shown in the Figure 5(b). 


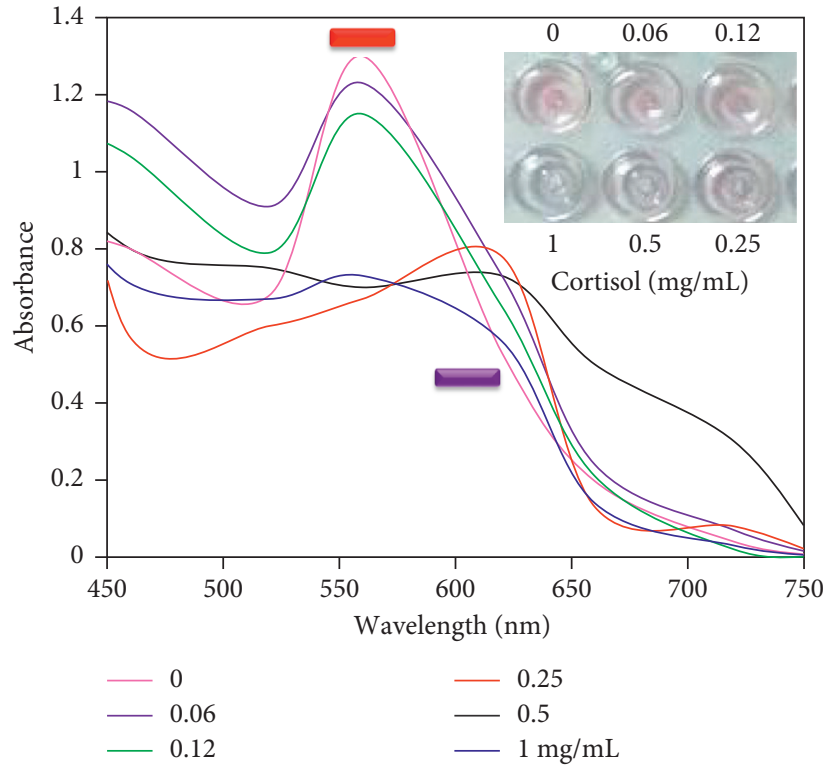

(a)

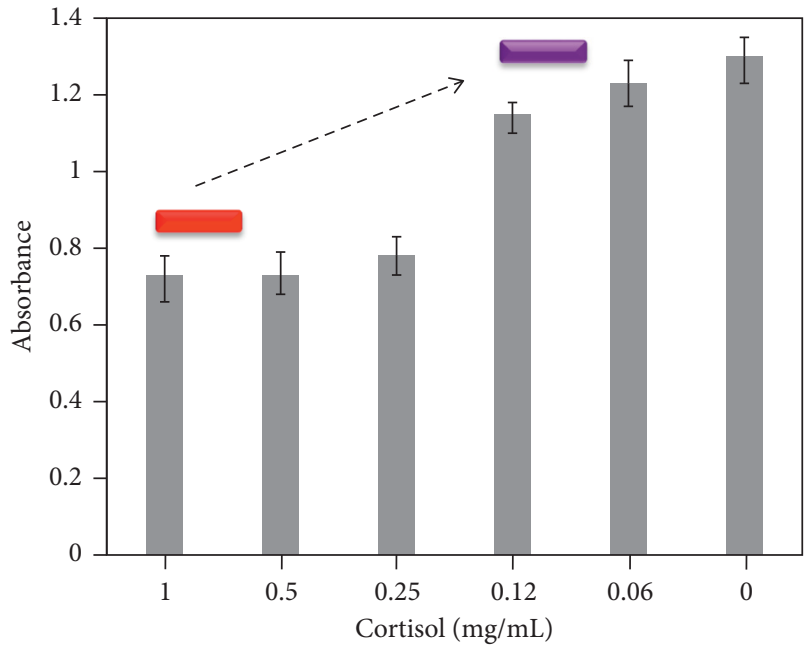

(b)

FIgURE 6: (a) Limit of detection with cortisol. Cortisol concentrations from 0 to $1 \mathrm{mg} / \mathrm{mL}$ were mixed independently with GNR-aptamer conjugates and the aggregation was checked in the presence of $\mathrm{NaCl}$. Inset displays the color developments. (b) Peak absorbance maximums with different concentrations of cortisol, averaged with different experimental replicates. The arrow indicates the direction of the changes.

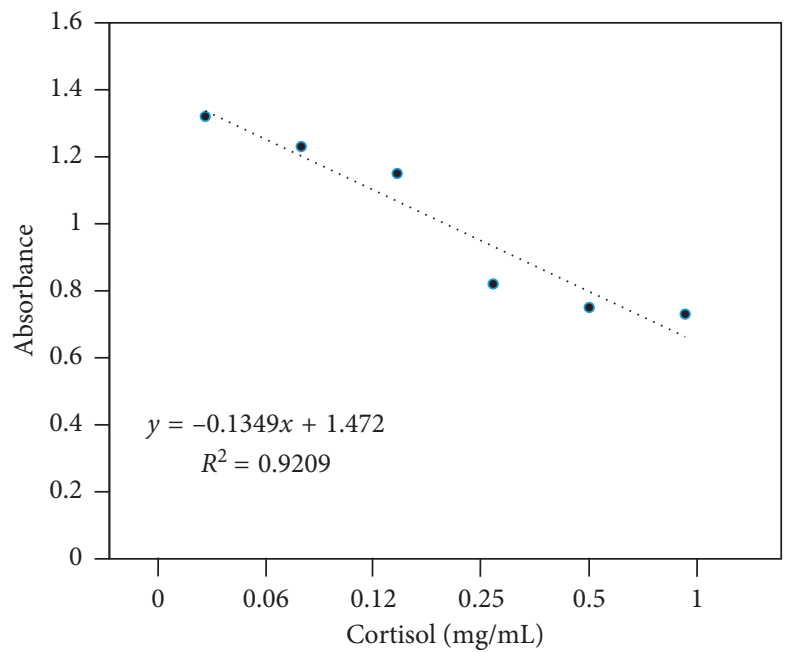

(a)

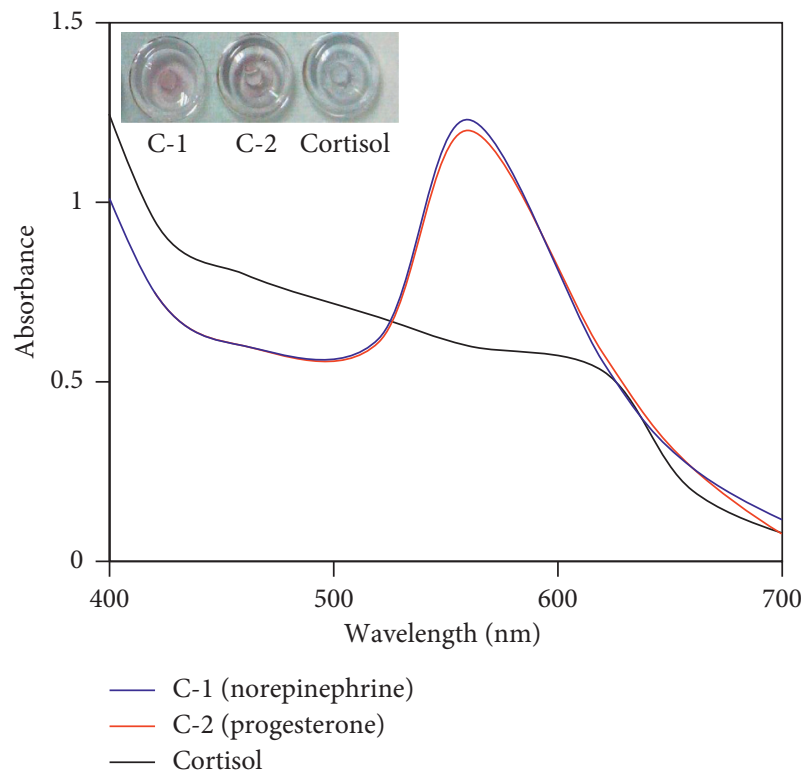

(b)

FIGURE 7: (a) Linear relationship between the absorption maximum and the concentration of the cortisol. 0 to $1 \mathrm{mg} / \mathrm{mL}$ of cortisol were used to detect by using GNR-aptamer conjugates. (b) Specific detection of cortisol was carried out with two different control hormones (norepinephrine (C1) and progesterone (C2)). The aptamers interacted with only cortisol, and the color of the GNR was changed to purple due to the aggregation.

\subsection{Determination of Limit of Detection with GNR Aggregation.} Since it was found that $1 \mathrm{mg} / \mathrm{mL}$ of cortisol was clearly detected by the colorimetric assay, to evaluate the limit of detection, the titration was performed with the cortisol from $1 \mathrm{mg} / \mathrm{mL}$ down to $0.06 \mathrm{mg} / \mathrm{mL}$ under similar experimental conditions. Figure 6 explains the results of the cortisol detection at different concentrations. It was noticed that the color of the solution clearly changed at three concentrations $(1,0.5$, and $0.25 \mathrm{mg} / \mathrm{mL})$ of cortisol and the corresponding shifts in optical absorbance were found to be $0.73,0.73$, and 0.78 , respectively, at $\sim 620 \mathrm{~nm}$. The concentrations from 0.12 to $0.06 \mathrm{mg} / \mathrm{mL}$ did not show the spectral change and still retain their peak maximums at $550 \mathrm{~nm}$, which indicated that these cortisol concentrations are not sufficient to release the 
TABLE 1: Comparison among the available methods for the quantitative cortisol detection.

\begin{tabular}{|c|c|c|c|c|}
\hline Method & Material & Probe & Sensitivity & Reference \\
\hline Chemiresistor & Graphene & Antibody & $10 \mathrm{pg} / \mathrm{mL}$ & [34] \\
\hline Integrated electrode & $\mathrm{MOS}_{2}$ & Antibody & $1 \mathrm{ng} / \mathrm{mL}$ & {$[35]$} \\
\hline Printed electrode sensor & Graphene & Antibody & $0.1 \mathrm{ng} / \mathrm{mL}$ & {$[36]$} \\
\hline Electrochemical sensor & Cofired ceramic & Antibody & $10 \mathrm{pg} / \mathrm{mL}$ & [37] \\
\hline Electrochemical impedance spectroscopy & Zinc oxide & Antibody & $1 \mathrm{ng} / \mathrm{mL}$ & {$[38]$} \\
\hline Piezoelectric immunosensor & Gold-coated surface & Antibody & $36 \mu \mathrm{g} / \mathrm{mL}$ & [39] \\
\hline RAMAN spectroscopy & - & Antibody & Human serum $(\mathrm{ng} / \mathrm{mL})$ & {$[40]$} \\
\hline Electrochemical impedance spectroscopy & Gold electrode & Antibody & $0.5 \mathrm{mg} / \mathrm{mL}$ & {$[41]$} \\
\hline
\end{tabular}

aptamer from GNR. Figure 7(a) shows the linear relationship between the absorption maximum and the concentration of the cortisol. From these results, it was concluded that the limit of detection was found at $0.25 \mathrm{mg} / \mathrm{mL}$ of cortisol using the colorimetric assay and that it is more suitable to monitor the gestational hypertension with the changes in cortisol levels.

3.6. Comparative Analysis and Specificity. Table 1 summarizes the quantitative detection of cortisol by different methods including the conventional strategies. The primary advantages of the current colorimetric method is the visual detection by naked eye, which is absent in other methods for cortisol detection. In addition, colorimetric method does not need any prior handling experiences and the special instruments, thereby making it appealing over other methods. Furthermore, to compliment the obtained results, it was compared with the interdigitated electrode sensor and a clear binding was noticed with the same concentration used in the colorimetric assay. However, interdigitated electrode sensor gives a clear response compared to the colorimetric assay (Supplementary information (available here)).

Specific detection of cortisol was shown by performing the experiment with two different control hormones namely, norepinephrine and progesterone. As shown in Figure 7(b), with the control hormones, the GNR did not show the changes in color and the absorbance peak maximums were stable at $550 \mathrm{~nm}$. At the same time with the cortisol, the aptamer was released from the GNR upon interaction, and the color of the GNR solution was changed to purple due to the aggregation in the presence of $\mathrm{NaCl}$. From these results, it was concluded that cortisol was specifically detected by the colorimetric assay with aptamer-GNR.

\section{Conclusion}

Gestational hypertension causes various health issues to the mother and baby during and after the period of pregnancy. Identifying the real condition of hypertension with a suitable biomarker is mandatory to treat properly. In this work, cortisol, known as the "stress hormone," was detected by the colorimetric assay using aptamer and gold nanorod conjugate as the primary tools. Cortisol was clearly detected by showing the color change of the gold nanorod solution turning to purple from red with monovalent salt, and the limit of detection was found as $0.25 \mathrm{mg} / \mathrm{mL}$. This method of detection has advantages over other methods to quantify the levels of cortisol with a higher specificity and helps to treat gestational hypertension.

$\begin{array}{ll}\text { Abbreviations } \\ \text { GNR: } & \text { Gold nanorod } \\ \text { nM: } & \text { Nanomolar } \\ \text { nm: } & \text { Nanometer } \\ \text { mM: } & \text { Millimolar } \\ \text { OD: } & \text { Optical density } \\ \mu l: & \text { Microliter } \\ \text { 11 } \beta- & 11 \beta \text {-Hydroxysteroid dehydrogenase type } 2 \\ \text { HSD2: } & \\ \text { SELEX: } & \text { Systematic Evaluation of Ligands by } \\ & \text { Exponential Enrichment } \\ \text { GNP: } & \text { Gold nanoparticle } \\ \text { RNA: } & \text { Ribonucleic acid } \\ \text { DNA: } & \text { Deoxyribonucleic acid. }\end{array}$

\section{Data Availability}

All the data are fully available without restriction.

\section{Conflicts of Interest}

The authors declare that they have no conflicts of interest.

\section{Authors' Contributions}

All the authors contributed to the preparation of the manuscript and discussion. All the authors read and approved the final manuscript.

\section{Supplementary Materials}

It includes the detection of cortisol on interdigitated electrode sensor, for the comparative study. It also includes brief method and the obtained results. Figure S1: the interaction of cortisol and aptamer on interdigitated electrode sensor. Reference for the described method is also provided. (Supplementary Materials)

\section{References}

[1] T. P. Kelder, M. E. Penning, H.-W. Uh et al., "Quantitative polymerase chain reaction-based analysis of podocyturia is a feasible diagnostic tool in preeclampsia," Hypertension, vol. 60, no. 6, pp. 1538-1544, 2012. 
[2] G. Poprawski, E. Wender-Ozegowska, A. Zawiejska, and J. Brazert, "Modern methods of early screening for preeclampsia and pregnancy-induced hypertension-a review," Ginekologia Polska, vol. 83, no. 9, pp. 688-693, 2012.

[3] L. A. Magee, A. Pels, M. Helewa et al., "Diagnosis, evaluation, and management of the hypertensive disorders of pregnancy: executive summary," Journal of Obstetrics and Gynaecology Canada, vol. 30, no. 3, pp. S1-S2, 2014.

[4] K. Kosicka, A. Siemiątkowska, A. Szpera-Goździewicz, M. Krzyścin, G. H. Bręborowicz, and F. K. Główka, "Increased cortisol metabolism in women with pregnancy-related hypertension," Endocrine, vol. 61, no. 1, pp. 125-133, 2018.

[5] P. Vianna, M. E. Bauer, D. Dornfeld, and J. A. B. Chies, "Distress conditions during pregnancy may lead to preeclampsia by increasing cortisol levels and altering lymphocyte sensitivity to glucocorticoids," Medical Hypotheses, vol. 77, no. 2, pp. 188-191, 2011.

[6] P. Ferrari, "The role of $11 \beta$-hydroxysteroid dehydrogenase type 2 in human hypertension," Biochimica et Biophysica Acta (BBA)_Molecular Basis of Disease, vol. 1802, no. 12, pp. 1178-1187, 2010.

[7] K. Kosicka, A. Siemiątkowska, M. Krzÿscin et al., "Glucocorticoid metabolism in hypertensive disorders of pregnancy: analysis of plasma and urinary cortisol and cortisone," PLoS One, vol. 10, no. 12, Article ID e0144343, 2015.

[8] T. Lakshmipriya, M. Fujimaki, S. C. B. Gopinath, K. Awazu, Y. Horiguchi, and Y. Nagasaki, "A high-performance waveguide-mode biosensor for detection of factor IX using PEGbased blocking agents to suppress non-specific binding and improve sensitivity," The Analyst, vol. 138, no. 10, pp. 28632870, 2013.

[9] S. C. B. Gopinath, "Methods developed for SELEX," Analitical and Bioanaltical Chemistry, vol. 387, no. 1, pp. 171-182, 2006.

[10] T. Lakshmipriya, M. Fujimaki, S. C. B. Gopinath, and K. Awazu, "Generation of anti-influenza aptamers using the systematic evolution of Ligands by exponential enrichment for sensing applications," Langmuir, vol. 29, no. 48, pp. 15107-15115, 2013.

[11] S. Gopinath, Y. Shikamoto, H. Mizuno, and P. Kumar, "A potent anti-coagulant RNA aptamer inhibits blood coagulation by specifically blocking the extrinsic clotting pathway," Thrombosis and Haemostasis, vol. 95, no. 5, pp. 767-771, 2006.

[12] T. Lakshmipriya, Y. Horiguchi, and Y. Nagasaki, "Coimmobilized poly(ethylene glycol)-block-polyamines promote sensitivity and restrict biofouling on gold sensor surface for detecting factor IX in human plasma," The Analyst, vol. 139, no. 16, pp. 3977-3985, 2014.

[13] S. C. B. Gopinath, "Biosensing applications of surface plasmon resonance-based Biacore technology," Sensors and Actuators B: Chemical, vol. 150, no. 2, pp. 722-733, 2010.

[14] S. C. B. Gopinath, K. Awazu, and M. Fujimaki, "Waveguidemode sensors as aptasensors," Sensors, vol. 12, no. 2, pp. 2136-2151, 2012.

[15] Q. Wei, R. Nagi, K. Sadeghi et al., "Detection and spatial mapping of mercury contamination in water samples using a smart-phone," ACS Nano, vol. 8, no. 2, pp. 1121-1129, 2014.

[16] X. Ma, Y. Liu, N. Zhou, N. Duan, S. Wu, and Z. Wang, "SERS aptasensor detection of Salmonella typhimurium using a magnetic gold nanoparticle and gold nanoparticle based sandwich structure," Analytical Methods, vol. 8, no. 45, pp. 8099-8105, 2016.
[17] R. Xiao, D. Wang, Z. Lin et al., "Disassembly of gold nanoparticle dimers for colorimetric detection of ochratoxin A," Analytical Methods, vol. 7, no. 3, pp. 842-845, 2015.

[18] S. C. B. Gopinath, P. K. R. Kumar, and J. Tominaga, “A BioDVD media with multilayered structure is suitable for analyzing biomolecular interactions," Journal of Nanoscience and Nanotechnology, vol. 11, no. 7, pp. 5682-5688, 2011.

[19] S. C. B. Gopinath and P. K. R. Kumar, "Aptamers that bind to the hemagglutinin of the recent pandemic influenza virus H1N1 and efficiently inhibit agglutination," Acta Biomaterialia, vol. 9, no. 11, pp. 8932-8941, 2013.

[20] S. C. B. Gopinath, K. Awazu, M. Fujimaki et al., "Influence of nanometric holes on the sensitivity of a waveguide-mode sensor: label-free nanosensor for the analysis of RNA aptamer-ligand interactions," Analytical Chemistry, vol. 80, no. 17 , pp. 6602-6609, 2008.

[21] L. Chen, J. Li, and L. Chen, "Colorimetric detection of mercury species based on functionalized gold nanoparticles," ACS Applied Materials \& Interfaces, vol. 6, no. 18, pp. 15897-15904, 2014.

[22] C.-S. Tsai, T.-B. Yu, and C.-T. Chen, "Gold nanoparticlebased competitive colorimetric assay for detection of proteinprotein interactions," Chemical Communications, vol. 34, no. 34, pp. 4273-4275, 2005.

[23] H. Li and L. Rothberg, "Colorimetric detection of DNA sequences based on electrostatic interactions with unmodified gold nanoparticles," Proceedings of the National Academy of Sciences, vol. 101, no. 39, pp. 14036-14039, 2004.

[24] C. D. Medley, J. E. Smith, Z. Tang, Y. Wu, S. Bamrungsap, and W. Tan, "Gold nanoparticle-based colorimetric assay for the direct detection of cancerous cells," Analytical Chemistry, vol. 80, no. 4, pp. 1067-1072, 2008.

[25] Z. Hu, M. Xie, D. Yang et al., "A simple, fast, and sensitive colorimetric assay for visual detection of berberine in human plasma by $\mathrm{NaHSO}_{4}$-optimized gold nanoparticles," RSC Advances, vol. 7, no. 55, pp. 34746-34754, 2017.

[26] M. Sabela, S. Balme, M. Bechelany, J.-M. Janot, and K. Bisetty, "A review of gold and silver nanoparticle-based colorimetric sensing assays," Advanced Engineering Materials, vol. 19, no. 12, Article ID 1700270, 2017.

[27] L.-K. Chau, Y.-F. Lin, S.-F. Cheng, and T.-J. Lin, "Fiber-optic chemical and biochemical probes based on localized surface plasmon resonance," Sensors and Actuators B: Chemical, vol. 113, no. 1, pp. 100-105, 2006.

[28] J. H. An, D.-K. Choi, K.-J. Lee, and J.-W. Choi, "Surfaceenhanced Raman spectroscopy detection of dopamine by DNA Targeting amplification assay in Parkisons's model," Biosensors and Bioelectronics, vol. 67, pp. 739-746, 2015.

[29] Y. Li, H. J. Schluesener, and S. Xu, "Gold nanoparticle-based biosensors,” Gold Bulletin, vol. 43, no. 1, pp. 29-41, 2010.

[30] F.-a. Wang, T. Lakshmipriya, and S. C. B. Gopinath, "Red spectral shift in sensitive colorimetric detection of tuberculosis by ESAT-6 antigen-antibody Complex : a new strategy with gold nanoparticle," Nanoscale Research Letters, vol. 13, no. 1, pp. 1-8, 2018.

[31] S. C. B. Gopinath, T. Lakshmipriya, and K. Awazu, "Colorimetric detection of controlled assembly and disassembly of aptamers on unmodified gold nanoparticles," Biosensors and Bioelectronics, vol. 51, pp. 115-123, 2014.

[32] B. J. Sanghavi, J. A. Moore, J. L. Chávez et al., “Aptamerfunctionalized nanoparticles for surface immobilization-free electrochemical detection of cortisol in a microfluidic device," Biosensors and Bioelectronics, vol. 78, pp. 244-252, 2016. 
[33] C. Yu and J. Irudayaraj, "Multiplex biosensor using gold nanorods," Analytical Chemistry, vol. 79, no. 2, pp. 572-579, 2007.

[34] Y.-H. Kim, K. Lee, H. Jung et al., "Direct immune-detection of cortisol by chemiresistor graphene oxide sensor," Biosensors and Bioelectronics, vol. 98, pp. 473-477, 2017.

[35] D. Kinnamon, R. Ghanta, K.-C. Lin, S. Muthukumar, and S. Prasad, "Portable biosensor for monitoring cortisol in lowvolume perspired human sweat," Scientific Reports, vol. 7, no. 1, pp. 13312-13317, 2017.

[36] S. K. Tuteja, C. Ormsby, and S. Neethirajan, "Noninvasive label-free detection of cortisol and lactate using graphene embedded screen-printed electrode," Nano-Micro Letters, vol. 10, no. 3, p. 41, 2018.

[37] A. Yndart, R. Jayant, V. Sagar et al., "Electrochemical sensing method for point-of-care cortisol detection in human immunodeficiency virus-infected patients," International Journal of Nanomedicine, vol. 10, pp. 677-685, 2015.

[38] R. D. Munje, S. Muthukumar, and S. Prasad, "Interfacial tuning for detection of cortisol in sweat using $\mathrm{ZnO}$ thin films on flexible substrates," IEEE Transactions on Nanotechnology, vol. 16, no. 5, pp. 832-836, 2017.

[39] B. S. Attili and A. A. Suleiman, "A piezoelectric immunosensor for the detection of cortisol," Analytical Letters, vol. 28, no. 12, pp. 2149-2159, 1995.

[40] K. Gracie, S. Pang, G. M. Jones, K. Faulds, J. Braybrook, and D. Graham, "Detection of cortisol in serum using quantitative resonance Raman spectroscopy," Analytical Methods, vol. 9, no. 10, pp. 1589-1594, 2017.

[41] M. Pali, J. E. Garvey, B. Small, and I. I. Suni, "Detection of fish hormones by electrochemical impedance spectroscopy and quartz crystal microbalance," Sensing and Bio-Sensing Research, vol. 13, pp. 1-8, 2017. 

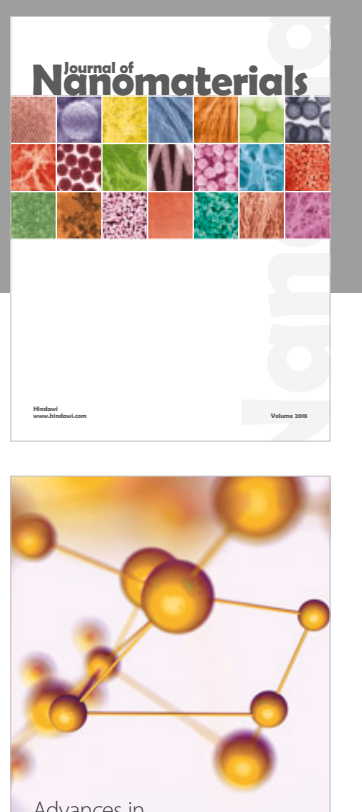

Physical Chemistry
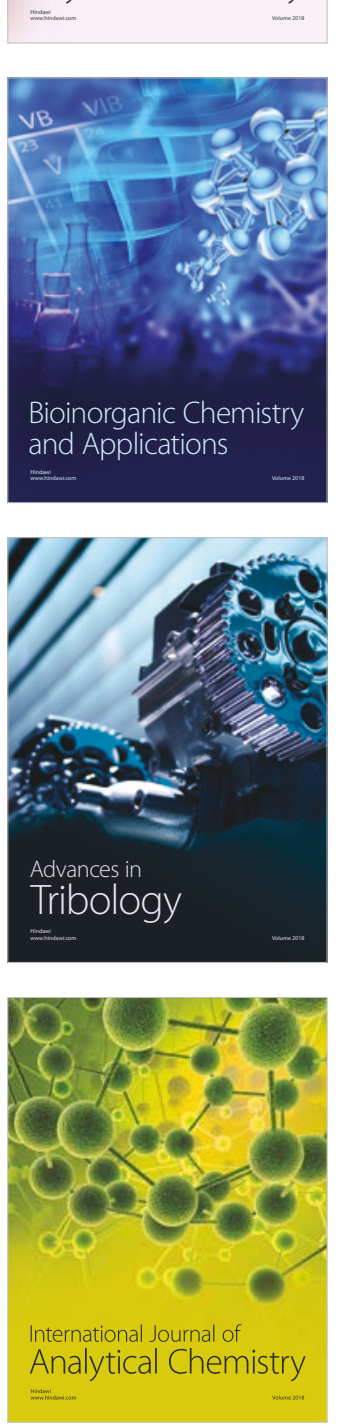

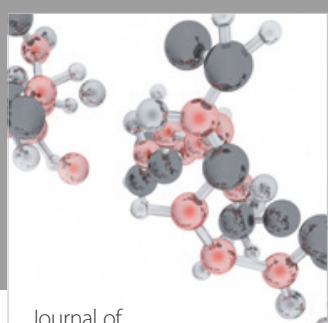

Analytical Methods

in Chemistry

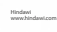

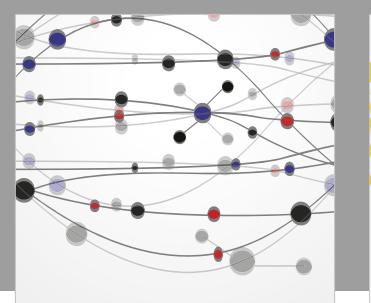

The Scientific World Journal

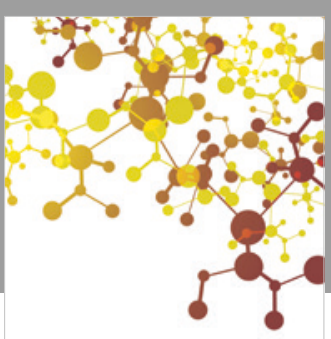

Journal of

Applied Chemistry
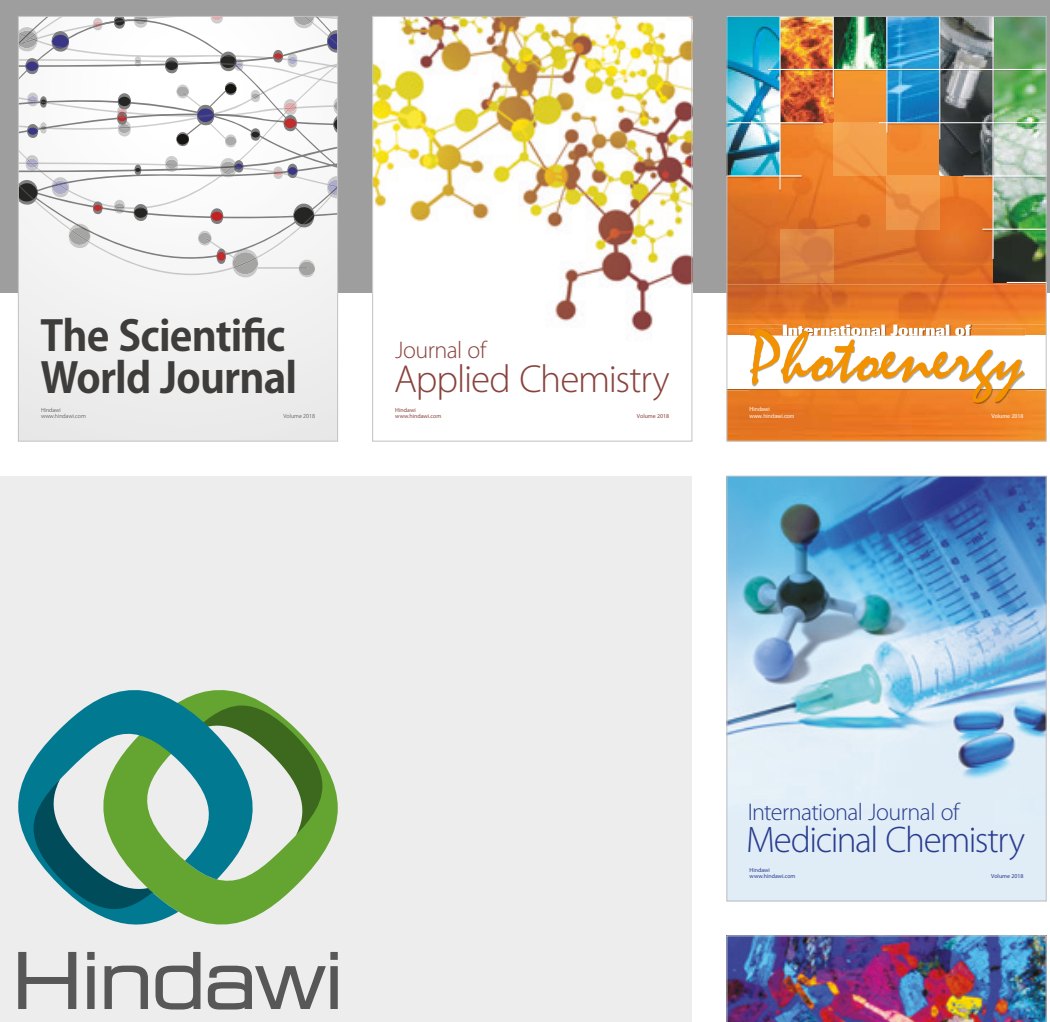

Submit your manuscripts at

www.hindawi.com
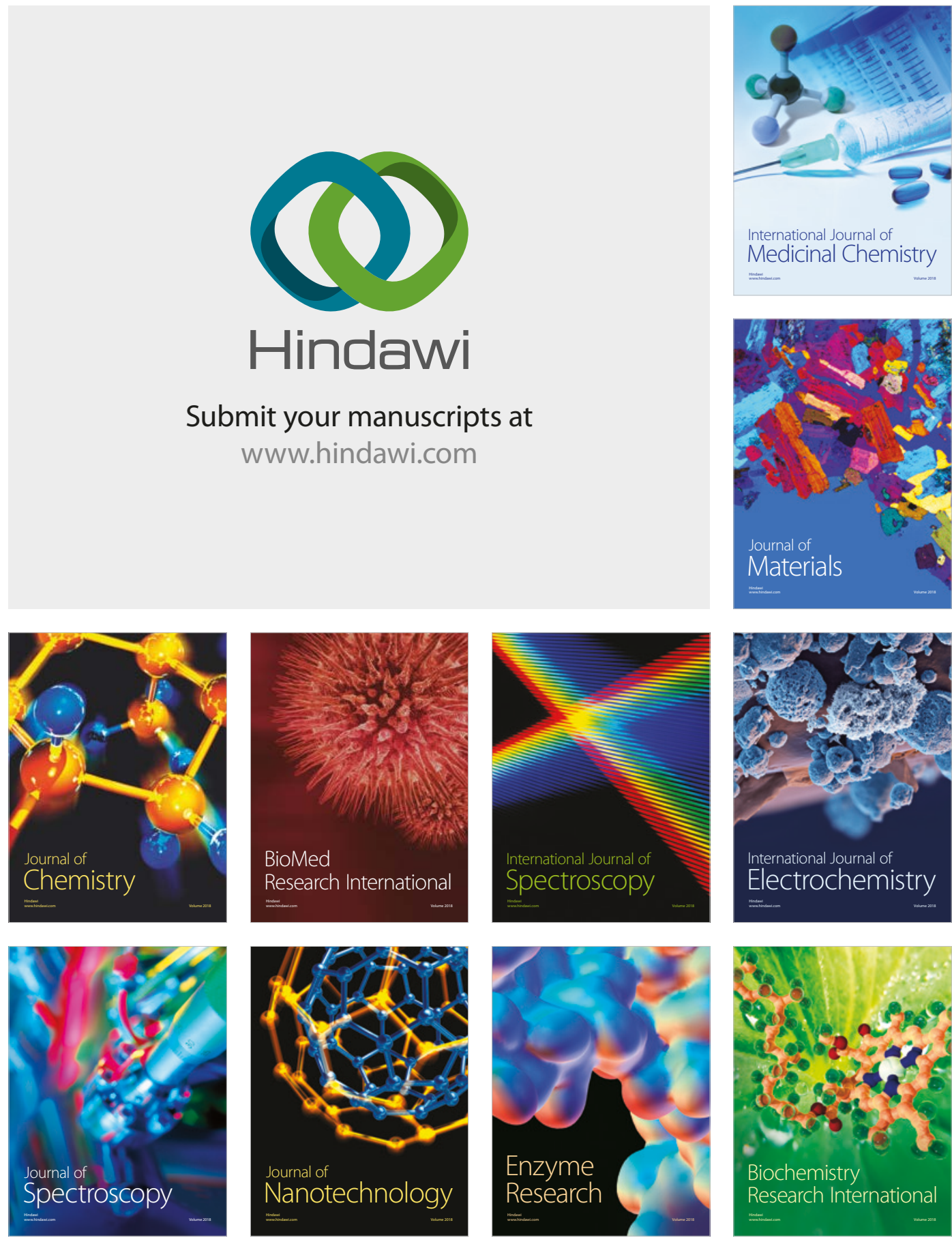
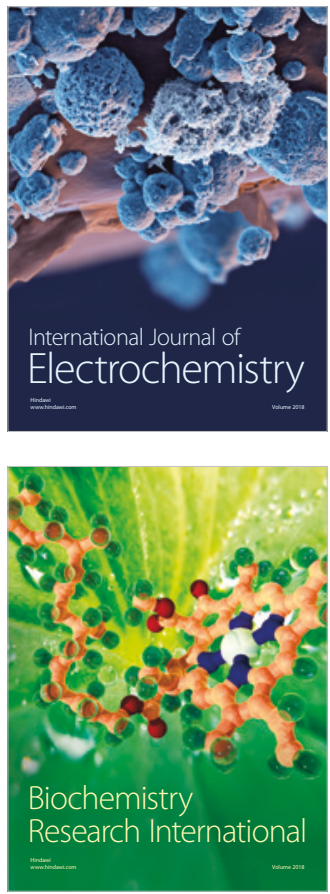